\title{
Electrophysiological Signatures of Intrinsic Functional Connectivity Related to rTMS Treatment for Mal de Debarquement Syndrome
}

\author{
Yoon-Hee Cha ${ }^{1,2}\left(\mathbb{0} \cdot\right.$ Guofa Shou $^{3} \cdot$ Diamond Gleghorn $^{1} \cdot$ Benjamin C. Doudican ${ }^{1} \cdot$ Han Yuan ${ }^{1,3,4} \cdot$ Lei Ding $^{1,3,4}$
}

Received: 25 February 2018 / Accepted: 7 August 2018 / Published online: 11 August 2018

(c) The Author(s) 2018

\begin{abstract}
To determine intrinsic functional connectivity (IFC) related to symptom changes induced by rTMS in mal de debarquement syndrome (MdDS), a motion perceptual disorder induced by entrainment to oscillating motion. Twenty right-handed women (mean age: $52.9 \pm 12.6$ years; mean duration illness: $35.2 \pm 24.2$ months) with MdDS received five sessions of rTMS (1 Hz right DLPFC, $10 \mathrm{~Hz}$ left DLPFC) over consecutive days. High-density (128-channel) resting-state EEG were recorded prior to and following treatment sessions and analyzed using a group-level independent component (IC) analysis. IFC between 19 ICs was quantified by inter-IC phase coherence (ICPC) in six frequency bands (delta, theta, low alpha, high alpha, beta, gamma). Correlational analyses between IFCs and symptoms were performed. Symptom improvement after rTMS was significantly correlated with (1) an increase in low alpha band (8-10 Hz) IFC but a decrease of IFC in all other bands, and (2) high baseline IFC in the high alpha (11-13 Hz) and beta bands (14-30 Hz). Most treatment related IFC changes occurred between frontal and parietal regions with a linear association between the degree of symptom improvement and the number of coherent IFC changes. Frequency band and region specific IFC changes correlate with and can predict symptom changes induced by rTMS over DLPFC in MdDS. MdDS symptom response correlates with high baseline IFC in most frequency bands. Treatment induced increase in long-range low alpha IFC and decreases in IFC in other bands as well as the proportion of coherent IFC changes correlate with symptom reduction.
\end{abstract}

Keywords rTMS · Mal de debarquement syndrome $\cdot$ Intrinsic functional connectivity $\cdot$ EEG $\cdot$ Inter-independent component phase coherence

Handling Editor: Carlo Miniussi.

Yoon-Hee Cha and Guofa Shou are co-first authors.

Yoon-Hee Cha

ycha@laureateinstitute.org

1 Laureate Institute for Brain Research, 6655 South Yale Avenue, Tulsa, OK 74136, USA

2 University of Tulsa, Tulsa, OK, USA

3 Stephenson School of Biomedical Engineering, University of Oklahoma, Norman, OK, USA

4 Institute for Biomedical Engineering, Science and Technology, University of Oklahoma, Norman, OK, USA

\section{Introduction}

Mal de debarquement Syndrome (MdDS) is a motion-triggered disorder of persistent oscillating vertigo that occurs after prolonged exposure to passive motion (Bisdorff et al. 2009; Brown and Baloh 1987). As opposed to motion sickness that occurs during the motion exposure, MdDS occurs after the motion stimulus has ended (Golding 2016). It represents the consequence of the human brain's entrainment to external oscillating motion such as during sea travel, the most common trigger (Cha 2009; Hain et al. 1999). The hallmark feature of MdDS includes a perception of rhythmic self-motion described as rocking, bobbing, or swaying that is only relieved with re-exposure to passive motion (Hain and Cherchi 2016; Hain et al. 1999; Cha et al. 2008; Cha 2009). While brief episodes of post-motion exposure dizziness and vertigo lasting less than two days are common, persistent episodes of MdDS lasting for months or years can occur in an important minority of individuals (Cha 2012; Hain et al. 
1999; Cha et al. 2008; Stoffregen et al. 2013; Gordon et al. 2000; Nachum et al. 2004).

Sensory entrainment to motion that does not revert back to the native state has neuroimaging signatures (Ding et al. 2014; Yuan et al. 2017; Cha et al. 2012). Functional neuroimaging of the MdDS brain state has revealed increased metabolism in the left entorhinal cortex and amygdala, two brain areas that exhibit entrainment to periodic electrical stimulation (Cha et al. 2012; Egorov et al. 2002, 2006; Fransén et al. 2006). Enhanced functional connectivity between the entorhinal cortex and posterior parietal and occipital areas that serve visual, vestibular, and somatosensory integration functions has been noted in MdDS (Cha et al. 2012).

Repetitive transcranial magnetic stimulation (rTMS) over the dorsolateral prefrontal cortex (DLPFC) is able to acutely reduce the perception of oscillating self-motion in MdDS (Cha et al. 2013). Connectivity between the entorhinal cortex and the posterior default mode network decreases with symptom improvement after rTMS over DLPFC (Ding et al. 2014; Yuan et al. 2017; Cha et al. 2012). Laterality plays a role in the optimal treatment paradigm in MdDS with righthanded subjects responding better to left DLPFC $10 \mathrm{~Hz}$ stimulation and left-handed subjects responding better to right DLPFC $10 \mathrm{~Hz}$ stimulation. Additionally, right $1 \mathrm{~Hz}$ stimulation in right-handed subjects also confers benefits (Cha et al. 2013). These findings are consistent with rTMS studies over the DLPFC in depression in which both high frequency $(10 \mathrm{~Hz})$ over the left DLPFC and low frequency $(1 \mathrm{~Hz})$ over the right DLPFC confer anti-depressant effects (Liu et al. 2017; Donse et al. 2018). The parallel is relevant because depression scores are higher in MdDS than in healthy controls and there may be synergistic benefit to both motion perception and mood obtained by targeting a shared circuitry (O'Reardon et al. 2007; Liao et al. 2011; Arroll et al. 2014).

Resting-state intrinsic functional connectivity (IFC) is a term used to denote baseline connectivity that is not stimulus driven (Van Dijk et al. 2009). It may be quantified as inter-independent component phase coherence (ICPC) on high-density EEG, which has previously been shown to correlate with symptom status in MdDS after bilateral DLPFC stimulation (Ding et al. 2014). Posterior predominant ICPC reductions occur in individuals with MdDS who are successfully treated with rTMS over the DLPFC (Shou et al. 2014; Ding et al. 2014). A model for MdDS has been proposed in which limbic oscillators that have widespread functional connectivity are tuned by the periodic stimulation of passive motion exposure that, in the presence of a relative reduction of prefrontal control over these limbic areas, causes a maladaptive physiological state to become persistent (Dickson et al. 2000; Cha 2015). Notably, MdDS may recur spontaneously after remission, indicating a training effect or memory of a maladaptive state in some cases. In the right physiological background, this might occur spontaneously without a motion trigger (Cha et al. 2008, 2018; Cha and Cui 2013).

MdDS represents a natural model of sensory entrainment to environmental motion that may provide new insights into the human brain's strategy for encoding periodic motion. We present IFC changes in a larger group of MdDS patients with a more thorough analysis method than our prior studies using the same rTMS paradigm (Ding et al. 2014; Shou et al. 2014). We have additionally determined baseline IFC characteristics that correlate with symptom improvement. We also present novel findings regarding the characterization of the IFCs as predictors of treatment effects, as well as the IFCs that simultaneously act as predictors and indicators of treatment effects.

\section{Materials and Methods}

\section{Informed Consent}

Study procedures were completed according to Declaration of Helsinki guidelines and approved by Western IRB (http:// www.wirb.com). Participants provided written informed consent and were recruited under ClinicalTrials.gov study NCT02470377. Details of the long-term treatment effects have been previously reported (Cha et al. 2016). This study used rTMS in an off-label manner (not used for the currently FDA approved indications for depression or peripheral nerve stimulation).

\section{Inclusion and Exclusion Criteria}

Inclusion criteria included: (1) chronic perception of rocking vertigo that started within two days after disembarking from sea, air, or land based travel; (2) symptoms lasting at least 6 months; (3) no other cause for symptoms after evaluation by a neurologist or otolaryngologist with appropriate testing for peripheral inner ear or other central nervous system cause for symptoms. Exclusion criteria included: (1) an unstable medical or psychiatric condition, including a history of bipolar disorder or psychosis; (2) pregnant or planning to become pregnant during the study; (3) contraindications to receiving rTMS or MRI, including medications known to reduce seizure threshold; (4) an unclear history of the onset of symptoms; (5) an inability to complete all study related testing.

\section{MRI Imaging}

All participants underwent structural brain MRI imaging for use in neuronavigation during the rTMS sessions. A T1-weighted magnetization-prepared rapid gradient-echo 
(MPRAGE) sequence with SENSE using the following parameters was acquired: $F O V=240 \mathrm{~mm}$, axial slices per $\mathrm{slab}=190$, slice thickness $=0.9 \mathrm{~mm}$, image matrix $=256 \times 256, \mathrm{TR} / \mathrm{TE}=5 / 2.012 \mathrm{~ms}$, acceleration factor $\mathrm{R}=2$, flip angle $=8^{\circ}$, inversion time $\mathrm{TI}=725 \mathrm{~ms}$, sampling band-width $=31.2 \mathrm{kHz}$.

\section{rTMS Procedures}

rTMS was performed with the Magventure MagPro X100 stimulator with a cooled figure-of-eight coil in biphasic mode with the handle back at a 45-degree angle relative to the mid-sagittal plane. The Localite TMS Navigator (Localite GmBH, Germany) frameless stereotaxy system was used for neuronavigation to identify the center of the DLPFC as the anterior portion of the middle of the middle frontal gyrus. Motor thresholds (MT) were determined each day with independent measurements made for both the right and left M1 hand areas before each of five treatment sessions that were performed on consecutive days at the same time each day. MTs were defined as the percent intensity of the stimulator output that generated a $50 \mu \mathrm{V}$ motor evoked potential in the contralateral abductor pollicis brevis muscle in $50 \%$ of trials. rTMS sessions consisted of $1 \mathrm{~Hz}$ right DLPFC stimulation at $110 \%$ of MT for 1200 pulses followed by $10 \mathrm{~Hz}$ left DLPFC stimulation at $110 \%$ MT for 2000 pulses. The $10 \mathrm{~Hz}$ protocol was administered as trains of 40 pulses over $4 \mathrm{~s}(10 \mathrm{~Hz})$ followed by $26 \mathrm{~s}$ of rest.

\section{Symptom Measures}

The participants rated their symptom status on a visual analogue scale (VAS) of 0-100 in which 0 represented complete absence of rocking vertigo and 100 represented symptoms so severe that they could not remain upright. The change of the VAS score from the first (Day 1) to the last (Day 5) day was calculated. Participants whose symptoms decreased by 10 -points or more were considered to be "positive" responders (improved); those whose scores increased by 10 points or more were considered "negative" responders (worsened); those with scores in-between were considered to be "neutral." The minimum criterion of 10 points was chosen because it best correlated with the participants' own perceptions of significant symptom change.

\section{EEG Recording and Preprocessing}

Two sessions of 5-min resting state EEG data acquisition were performed before the first rTMS session (Day 1) and about $3 \mathrm{~h}$ after the last rTMS session (Day 5) using a 128-channel EEG cap (Brain Products GmbH, Munich, Germany). The post rTMS EEG data were acquired with a delay because of the obligatory time spent to prepare the cap on the subject with electrode paste. The post-treatment EEG data thus represented more sustained neuromodulatory effects than if the recordings were made immediately following the last treatment. The online reference channel was located at the $\mathrm{FCz}$ position and the ground electrode at the $\mathrm{AFz}$ position. Impedance of all electrodes was maintained below $10 \mathrm{~K} \Omega$ with a sampling frequency of $1000 \mathrm{~Hz}$, an analog filter from 0.016 to $250 \mathrm{~Hz}$, and a resolution of $0.1 \mu \mathrm{V}$. Participants sat in a chair with their eyes closed in a quiet darkened room during the recording.

The preprocessing steps for each participant were fully detailed in Ding et al. 2014. To briefly review, data were sequentially processed as follows: (1) notch filtered at $60 \mathrm{~Hz}$, (2) band-pass filtered at $0.5-100 \mathrm{~Hz}$, (3) noisy channels removed, (4) segmented into one second epochs without overlap from continuous recordings, (5) noisy segments removed, (6) decomposed remaining data into 64 ICs via independent component analysis (ICA) implemented in EEGLAB (Delorme and Makeig 2004), (7) noisy ICs removed using the FASTER toolbox and visual inspection (Nolan et al. 2010). An average of 284 epochs were left after preprocessing for each recording session, which were further down-sampled to $250 \mathrm{~Hz}$ and re-referenced to a common average.

\section{Detection of IFCs at the Component Domain}

IFCs were measured at the component domain rather than the channel domain in order to reduce volume conduction effects on channel signals. First, a group-level ICA using a complex ICA model with a real-value mixing matrix was performed on the preprocessed EEG data that were concatenated across all sessions (Ding et al. 2014; Bingham and Hyvärinen 2000; Shou et al. 2012). The input data, complex values corresponding to $2-50 \mathrm{~Hz}$ with a resolution of $1 \mathrm{~Hz}$, was obtained by short-time Fourier transform. Second, brain activity related components were selected from all obtained components based on their spatio-spectral patterns for subsequent analysis (Ding et al. 2014). Third, IFC between different components, quantified as ICPC (Delorme and Makeig 2004), which is also known as phase locking value (Lachaux et al. 1999), was measured at six different frequency bands, i.e., delta $(2-3 \mathrm{~Hz})$, theta (4-7 Hz), low alpha $(8-10 \mathrm{~Hz})$, high alpha $(11-13 \mathrm{~Hz})$, beta $(14-30 \mathrm{~Hz})$, and gamma $(31-50 \mathrm{~Hz})$. The motivations for using ICPC were (1) phase coherence takes nonlinearity into account (Van Diessen et al. 2015), and (2) the calculation of phase coherence between independent components rather than channels has been shown to largely reduce the volume conduction effect in the estimation of functional connectivity (Shou and Ding 2015). 


\section{Identification of Treatment-Related IFC.}

To identify rTMS treatment-related IFCs, four different analyses were sequentially performed on ICPC values between different components.

1. Pre- and post-rTMS ICPC values were separately examined via a one-sample $t$ test to determine whether they were significantly different from zero (null hypothesis). The significance level was set to $p<0.05$ with Bonferroni correction [significance level divided by the number of IC pairs based on 19 selected ICs (i.e., $19 *(19-1) / 2$ ) * the number of frequency bands (i.e., 6)].

2. Cross-subject Pearson correlation analysis was performed on the pre- to post-rTMS (i.e., post-rTMS minus pre-rTMS) ICPC differences and VAS scores to identify symptom change-related IFCs. An IFC was of statistical significance only when the $p$ value of the correlation analysis was $<0.05$ and the ICPC value was significantly different from zero in either the pre- or post-rTMS session, as detected in the first analysis.

3. An independent cross-subject Pearson correlation analysis was performed between pre-rTMS (i.e. baseline) ICPC values and VAS score changes to identify IFCs whose baseline values correlated with treatment response. Here, an IFC was considered statistically significant only when the $p$ value of the correlation analysis was $<0.05$ and the ICPC value was significantly different from zero in the pre-rTMS session detected in the first analysis.

4. Based on the overlapping IFCs that showed consistently significant correlations in both the prior correlation analyses, we calculated the proportion of IFCs that exhibited the same direction (coherency) of changes induced by rTMS as indicated in the prior correlation analyses for each participant. A third cross-subject correlation analysis was then conducted between this proportion and the VAS score changes. The goal of this analysis was to examine the treatment-related IFC changes beyond individual IFCs in order to determine whether there was a general pattern of IFC changes that correlated with treatment response.

In steps 2 and 3, we did not perform a correction for multiple comparisons in terms of the number of IC pairs and frequency bands, but only implemented a constraint that the ICPC values were different from zero in either pre- or postrTMS session.

\section{Results}

\section{Demographic Information}

Twenty right-handed individuals with MdDS completed all 5 days of the protocol. Despite being open to either sex, only female participants volunteered for the study, reflecting the significantly higher proportion of women to men in this disorder (Cha et al. 2018; Mucci et al. 2018). Mean age at the time of the study was $52.9 \pm 12.6$ years, median of 56.5 years, and a range of $28-68$ years. The duration of illness had a mean of $35.2 \pm 24.2$ months, median 30 months, and a range of 8-96 months. Inciting triggers included water-based travel in 11, air travel in four, and land-based (car, train) travel in five. Changes of VAS scores from Day 1 to Day 5 are presented in Fig. 1.

\section{Components of Interest}

Nineteen ICs were selected based on their spatial and spectral characteristics (labeled as IC $k, k=1 \ldots 19$, Fig. 2). These components generally replicated the IC patterns of many other studies as well as our previous studies using restingstate EEG signals (Ding et al. 2014; Yuan et al. 2012; Chen et al. 2013). These ICs are displayed on a cortex model based on the dominant weights of their spatial patterns in Fig. 2A. Most of the ICs are located symmetrically on both hemispheres or along the midline of the cortex well-representing most brain regions, i.e., ICs 1-4 for occipital, ICs 7-10 for parietal, ICs 5-6, 11-14 for tempo-occipital/temporal, ICs 15-16 for motor, and ICs 17-19 for frontal regions. IC spectral patterns (Fig. 2B) followed the 1/f distribution in their power spectra with peaks at corresponding frequency bands,

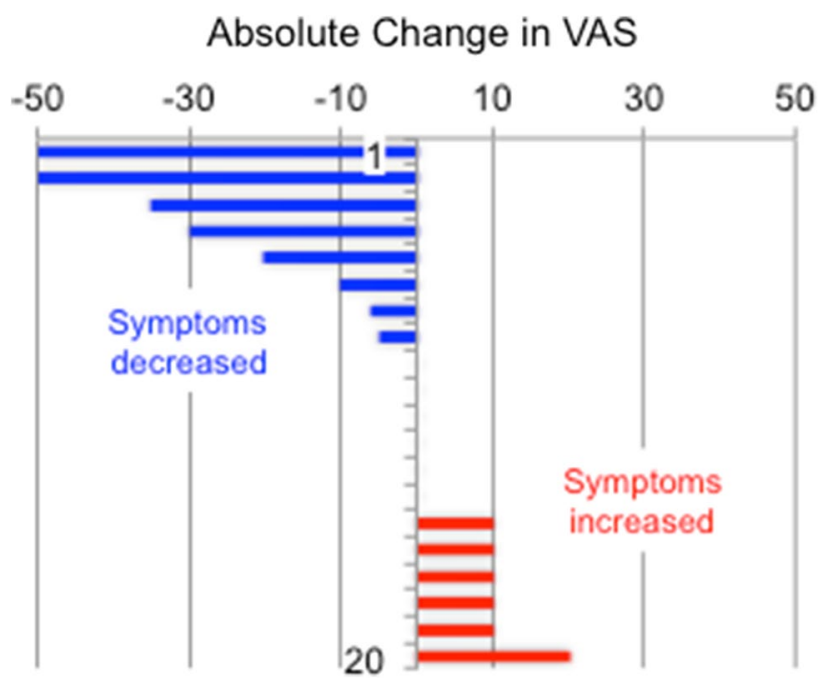

Fig. 1 Change in visual analogue scores (VAS) following rTMS 


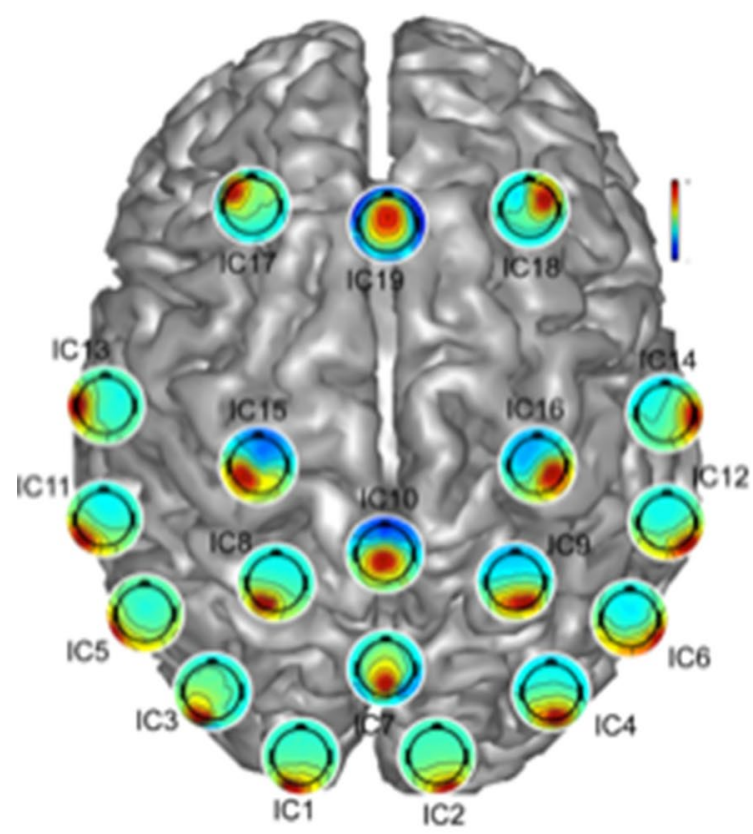

(A)
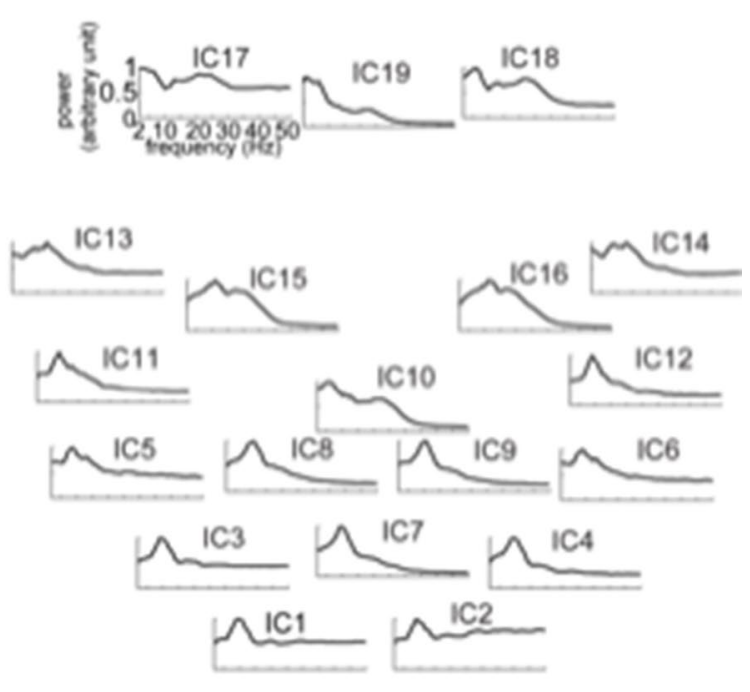

(B)

Fig. 2 Spatial and spectral patterns of 19 selected independent components arranged over the cortex according to the approximate locations of their neural substrates: A spatial patterns, B normalized grand averaged power spectra from 2 to $50 \mathrm{~Hz}$

i.e., peaks at alpha band for most ICs, and additional peaks in theta and beta bands for the ICs over frontal and parietal regions. Such phenomena were consistent with the regional specificity of the dominant frequency band power over different brain regions (Shou et al. 2012; Klimesch 1999).

\section{IFC as an Indicator of Treatment Effect}

Intrinsic functional connectivity changes that significantly correlated with VAS score changes are illustrated in Fig. 3. In the low frequencies (i.e., delta, theta, and low alpha), there were 6, 7, and 7 IFCs identified, respectively. In the high frequencies (i.e. high alpha, beta, and gamma), there were 11,11 , and 10 identified. The IFC changes in the low alpha band were all negatively correlated (positive vs. negative $=0$ vs. 7 ), indicating that rTMS-induced IFC enhancement in this band was correlated with a reduction in symptoms. Conversely, the IFCs in the high alpha and beta bands were predominantly positive (positive vs. negative $=9$ vs. 2 for high alpha, and 8 vs. 3 for beta), indicating a general rTMS-induced IFC reduction in the high alpha and beta bands with improvement in symptoms. The IFCs in other frequency bands showed no predominant directional correlation with symptom change. When counting the ICs that were identified in these IFCs across all frequency bands, six ICs were present at least once in five frequency bands, i.e., IC2, IC3, IC9, IC16, IC 18 and IC19, and five ICs were present in at least eight IFCs across all frequency bands, i.e., IC2(11), IC3(8), IC7(8), IC9(10), and IC18(8). It was notable that most of the ICs and IFCs that correlated with symptom changes were longrange and inter-hemispheric, especially related to frontal and parietal regions.

\section{IFC as a Predictor of Treatment Effect}

Baseline IFC measurements that were significantly correlated to VAS score changes and may be predictive of treatment response are illustrated in Fig. 4. Among the six frequency bands, 9, 13, and 13 IFCs were identified in the delta, high alpha, and beta bands, respectively. There were five IFCs identified in each of the theta, low alpha, and gamma bands. Most of the detected IFCs had negative correlations, indicating that, in general, high pre-rTMS functional connectivity was correlated with treatment response to rTMS. Regarding the involved ICs, four ICs were present at least once in five or more frequency bands, i.e., IC1, IC 3 and IC19 in five bands, and IC17 in six bands. Five ICs were present in 8 or more IFC measurements in all six-frequency bands, i.e., IC1, IC9, IC17 in 8 IFCs, IC3 in 9 IFCs, and IC19 in 10 IFCs. Similarly, these ICs were mainly in the frontal and posterior (parietal and occipital) regions, and most of the identified measures of IFC were involved in long-range and inter-hemispheric connections. 

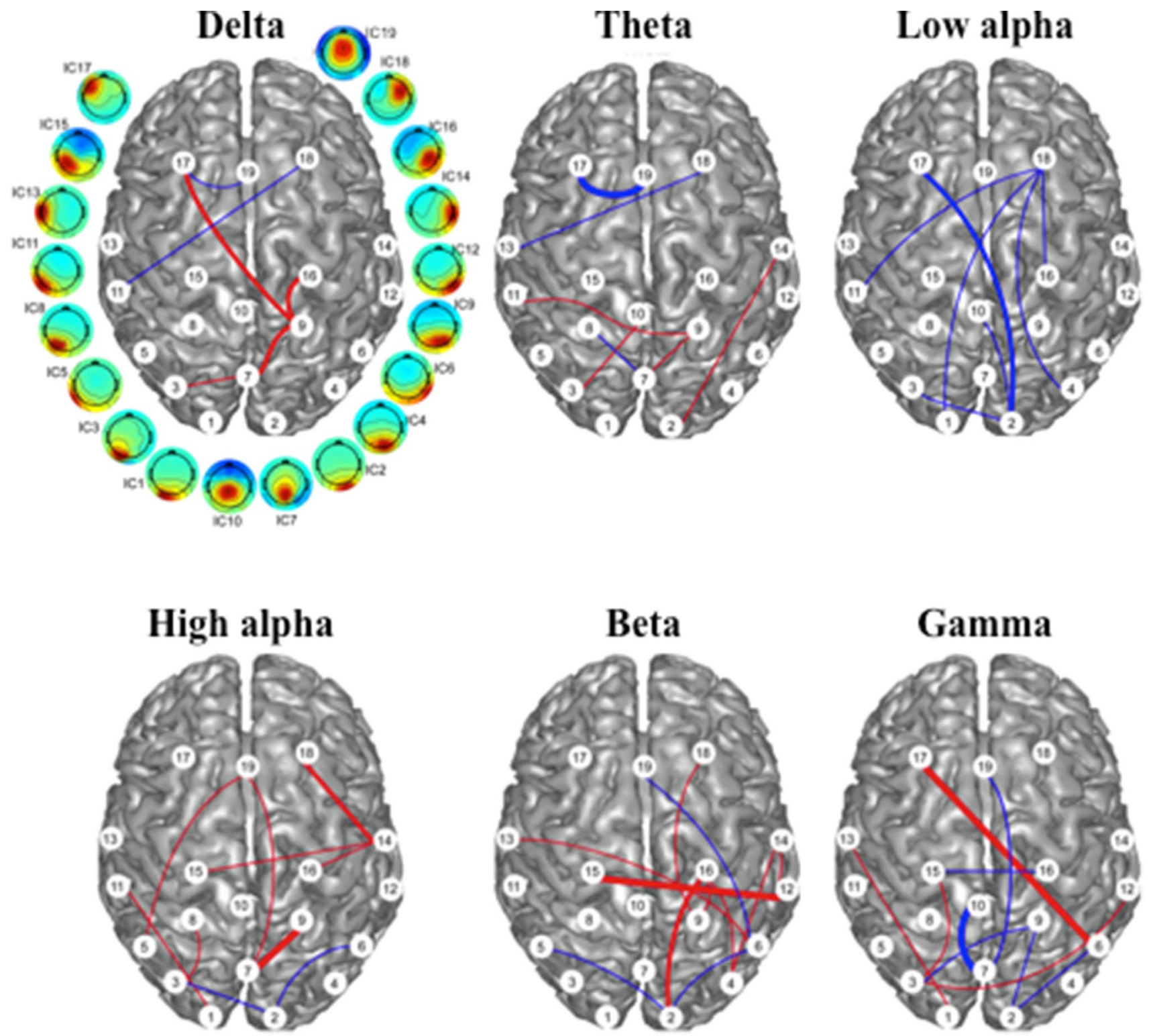

Fig. 3 Intrinsic functional connectivity changes correlated to VAS score change following rTMS. Red and blue lines indicate positive and negative correlations, respectively. Red: connectivity decreases with symptom improvement; blue: connectivity increases with symp-

\section{IFC with Both Predictive and Dynamic Changes Related to Treatment Effect}

A group of IFCs were identified with significant correlations in the preceding two types of correlation analyses. Among them, 16 IFCs were simultaneously identified with consistent correlation patterns (Fig. 4), i.e., they both changed with treatment response and were also predictive of treatment response. Among different frequency bands, most were detected in the high alpha and beta bands (i.e., 5), while no common IFCs were detected in the low alpha band. It is also tom improvement. The weight of the lines represents levels of significance: heavy: $p<0.005$, medium: $p<0.01$, light: $p<0.05$. White circles with numbering indicate the ICs noted in Fig. 2

notable that most of these common IFCs (14 out of 16) had high pre-rTMS values and negative changes (i.e. connectivity went down) corresponding to lowering VAS (symptom improvement).

The correlation analysis between the coherency of IFCs changes (exhibiting the same direction of change) and VAS score changes revealed a significantly negative relationship ( $r=-0.81, p=0.000013$, Fig. 5). This indicated that individuals with more IFCs exhibiting the same direction of change induced by rTMS (i.e., usually a positive correlation signifying a reduction in IFC with a reduction 

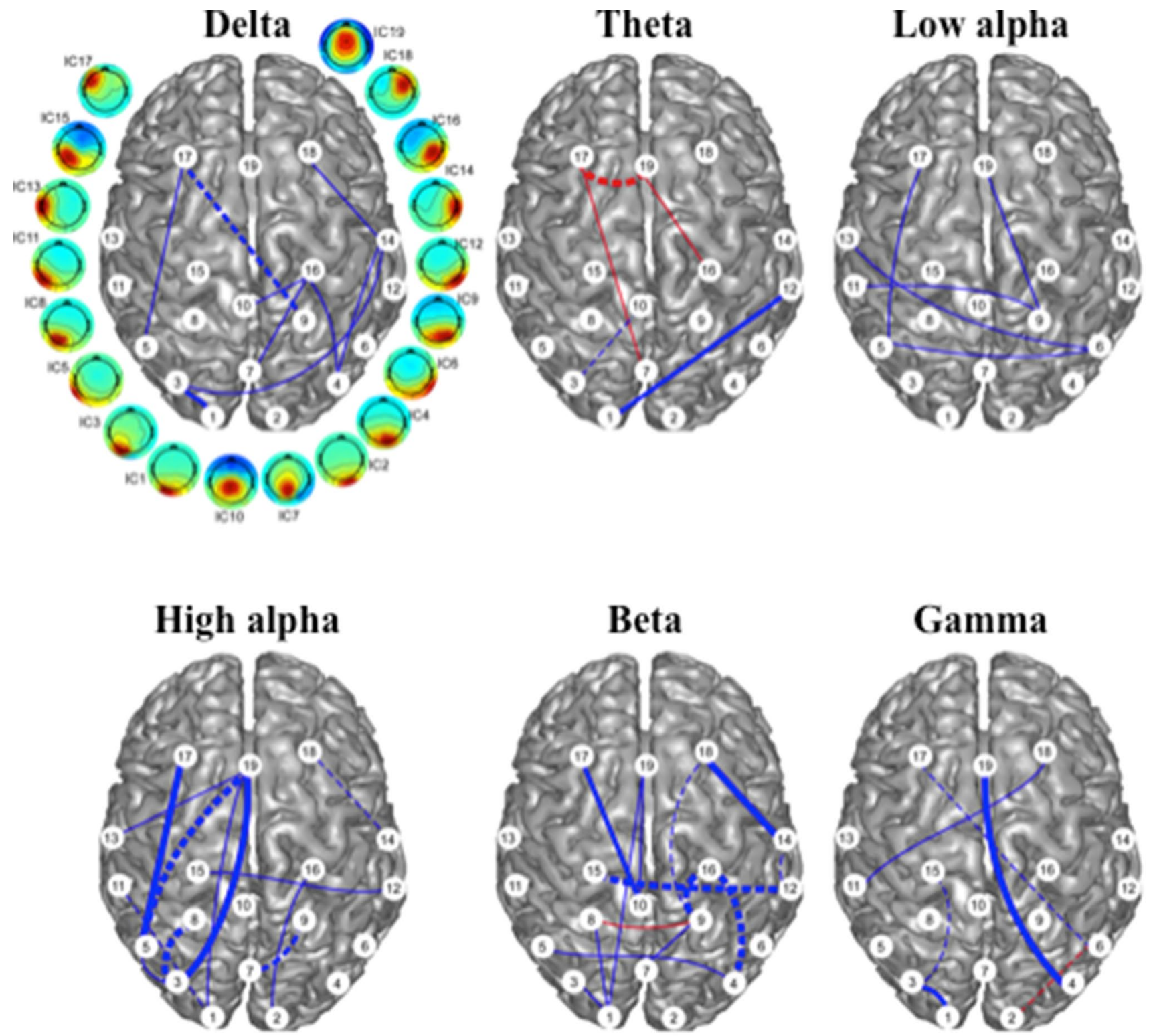

Fig. 4 Intrinsic functional connectivity (IFC) with baseline values significantly correlated with VAS score changes following rTMS. Red: low baseline connectivity correlated with symptom improvement; blue: high baseline connectivity correlated with symptom improvement. Dashed lines indicate IFCs that were identified in both the symptom correlation analysis shown in Fig. 3 and in the baseline correlation analysis. The weight of the lines represents levels of significance: heavy: $p<0.005$, medium: $p<0.01$, light: $p<0.05$. White circles with numbering indicate the ICs noted in Fig. 2

\section{Discussion}

The present study investigated electrophysiological signatures of rTMS treatment-related IFCs in individuals with MdDS by examining pre- and post-rTMS restingstate EEG signals and their correlation to symptom change. These findings provide a detailed evaluation of IFC changes induced by rTMS beyond those revealed in is a relevant factor in the pathogenesis of MdDS and specifically that de-synchronization of IFCs correlate with symptom improvement. 


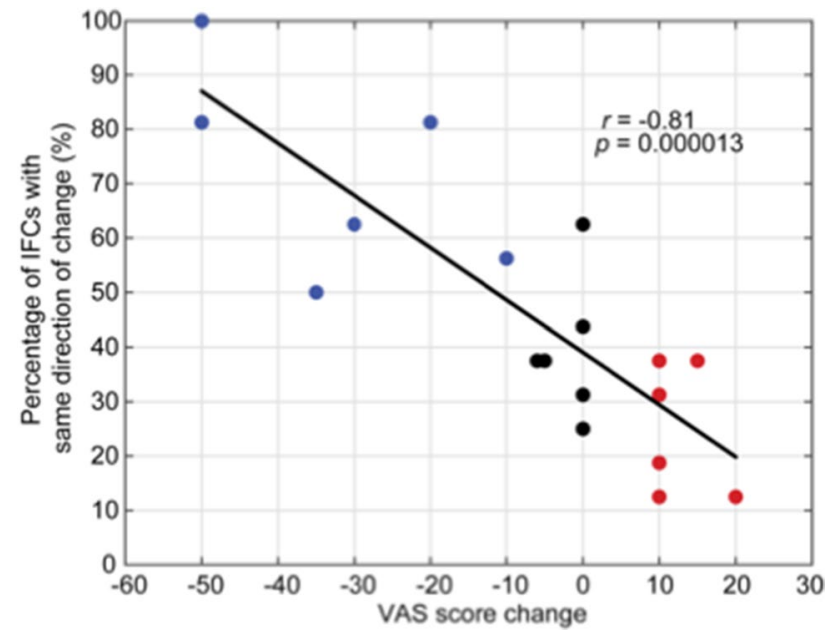

Fig. 5 Relationship between all simultaneously detected IFCs across all frequency bands (dashed lines in Fig. 4) and treatment effects for all subjects: blue, black and red dots represent positive, neutral and negative responders, respectively

a pilot study (Ding et al. 2014). Frequency- and regionspecific IFCs were found to be modulated after rTMS with changes significantly correlated to symptom change, especially in the low/high alpha and beta bands in the frontal and posterior regions. In addition, IFCs that predicted an individual's response to rTMS were identified, with high baseline values predictive of a positive treatment effect. More importantly, some IFCs that correlated with symptom change also overlapped with those predictive of symptom change.

The correlation analysis between IFC change and symptom change revealed a frequency band-dependent pattern (Fig. 3). Among six frequency bands, positive and negative correlations were dominant in the high alpha/beta bands, and low alpha, respectively. The different patterns for low and high alpha bands might be related to different neural mechanisms in these alpha frequency sub-bands (Klimesch 1999; Klimesch et al. 1993).

Alpha oscillation is traditionally referred to as an "idling rhythm," and its fluctuation indicates either disengagement (high power) or engagement (low power) of specific neurocognitive processes (Klimesch et al. 2007; Klimesch 1999). Lower alpha band synchronizations and de-synchronizations are widespread and reflect general demands on attention (Klimesch et al. 1998; Neuper and Pfurtscheller 2001). The negative correlation of the low alpha band to symptom change (i.e. connectivity goes up when symptoms go down) may represent the unloading of the attentional demand created by the motion perception following rTMS. In contrast, upper alpha band power has been reported to decrease over the motor cortex during physical movement, visual cortex during visual stimulation, and temporal cortex during auditory stimulation (Chatrian et al. 1959; Berger 1929; Niedermeyer 1990). It has been proposed that upper alpha amplitude is associated with the inhibition of nonessential cognitive processing, allowing greater efficiency of top-down control over the task at hand (Klimesch et al. 2007; Cooper et al. 2003; Bazanova and Vernon 2014). Therefore, the reduced connectivity of high alpha band following rTMS could be correlated with less rhythmic idling and better engagement of both cognitive and external sensory processing.

Compared to other frequency bands, the functional role of beta oscillations remains controversial (Engel and Fries 2010). The identified pattern of dominant positive correlation in the beta band might be interpreted by the proposed "status quo" hypothesis (Engel and Fries 2010), which suggests that beta activity is associated with the maintenance of the present sensorimotor or cognitive state with its reduction indicating a change of the status quo. Alternatively, a second recently proposed theory for the role of beta-synchronization suggests that it may mediate the transition from latent to active cortical representations, which may be content specific (Spitzer and Haegens 2017). According to this theory, the beta band may be a "transitional" rhythm that bridges the gap between alpha frequency, which is associated with top-down inhibition (Klimesch et al. 2007) and gamma frequency $(30-100 \mathrm{~Hz})$, which is associated with local activity (Whittingstall and Logothetis 2009). Changes in connectivity in the beta band, therefore, may reflect an alteration in the ability to reactivate latent endogenous activity.

The correlation analysis in this study on pre-TMS values revealed a pattern of mostly negative correlations among all six bands (Fig. 4), indicating that individuals with higher baseline values achieved better treatment outcomes. More importantly, some IFCs were detected in both correlation analyses indicating that they had both predictive and dynamic features (Fig. 4), i.e., treatment responders were individuals with high baseline IFC values that reduced following rTMS. These data were consistent with high baseline resting state functional connectivity between DLPFC and entorhinal cortex correlating with treatment response to rTMS in MdDS (Yuan et al. 2017). The DLPFC is also the main target for rTMS treatment of medically refractory depression, with a resultant large body of literature on the neuroimaging correlates of DLPFC stimulation for depression (Perera et al. 2016; Fischer et al. 2016). In particular, the therapeutic effect by targeted stimulation at DLPFC has been linked to a modulatory effect in the circuitry of the default mode network (Liston et al. 2014). These hubs include the frontal and posterior regions observed in the current study. Furthermore, Drysdale et al. (2017) reported that intrinsic network connectivity is predictive of TMS treatment response for depression with key regions involved in predicting responders including the DLPFC and posterior 
parietal cortex. This is again consistent with our observations, despite being based on different neuroimaging modalities. In contrast, anti-correlation between DLPFC and subgenual anterior cingulate cortex has been shown to predict treatment response to rTMS for depression, indicating that specific networks may exhibit oppositely directed dynamic changes related to symptom response (Fox et al. 2012).

To further examine the correlation between IFCs and treatment effect, we performed a third correlation analysis (Fig. 5). The results indicated that not only individual IFCs but also the proportion of dynamic IFC changes across all frequency bands is a marker of treatment response. Specifically, responders to rTMS exhibited a greater proportion of IFC de-synchronizations, regardless of frequency. Therefore, these common IFCs as well as the number of IFCs could be biomarkers used to both predict and monitor treatment effects. Thus, a goal of treatment for MdDS might be to induce a greater proportion of IFC de-synchronizations.

Past studies have shown that functional connectivity to latent drivers of neural oscillators such as the entorhinal cortex is altered between individuals with MdDS and healthy controls, which may explain the widespread IFC changes observed in this study (Cha et al. 2012; Yuan et al. 2017). The components involved in the detected IFCs were mainly in the frontal and posterior regions. Though we targeted the DLPFC in this study, we have shown that induced IFC changes were indeed widespread, and were directional for those in whom a beneficial clinical effect was induced. IC2, IC3, and IC7 in the occipital and posterior parietal regions and IC17 and IC18 in the prefrontal regions were the most commonly observed locations that exhibited symptom related connectivity changes. This is especially relevant because the target of the treatment was the prefrontal cortex in the region of ICs 17 and 18, whereas symptom relevant visual and motion processing areas are in the regions of ICs 2,3 , and 7 . This raises the possibility that some posterior nodes could serve as potential stimulation targets for future studies.

The current results of IFC based on EEG data are related to previous investigations in resting-state functional connectivity using fMRI, particularly with regard to the default mode network changes (Yuan et al. 2016; Raichle et al. 2001; Buckner et al. 2008). EEG can reveal frequency-specific connectivity changes with a stronger relationship between certain frequency bands and symptom changes than others. A decrease in resting state fMRI connectivity in the posterior default mode network that correlated with symptom improvement after DLPFC stimulation in individuals with MdDS (Yuan et al. 2017) was consistent with the decrease of EEG connectivity in the delta, high-alpha, beta band and gamma bands observed in this study, indicating a positive coupling relationship between the fMRI data and restingstate EEG. Notably, the positive coupling in the beta and gamma frequency bands has been observed from simultaneous EEG and fMRI resting-state data as well as local field potential and fMRI data in other human studies (Mukamel et al. 2005; Mantini et al. 2007; Laufs et al. 2003).

When examining regions where EEG connectivity is associated with positive coupling with fMRI data, our results show near-range connections in the parietal and occipital regions as well as long-range connections between the parietal/temporal cortices and the medial frontal regions, hub regions of the default mode network. Indeed, consistent spatial patterns between EEG and fMRI resting-state networks have been confirmed in recent studies that report a high concordance between the spatial and temporal dynamics of the default mode network (Yuan et al. 2012, 2013, 2016). Our current study reports an opposite trend in the low alpha frequency band as well as theta band in the frontal regions (Fig. 3), indicating that symptom reductions are associated with EEG connectivity increases in these lower frequency bands. Compared with fMRI findings, the increase of EEG connectivity in the low-alpha frequency band coincides with a decrease of fMRI connectivity, suggesting a negative coupling relationship. Our observation of the negative coupling in the low-alpha frequency band with resting state fMRI is consistent with other human brain studies (Mukamel et al. 2005; Laufs et al. 2003; Goldman et al. 2002; de Munck et al. 2007).

\section{Limitations}

Several study limitations merit comment. For the purposes of our analyses, though an even distribution of treatment responses created an input variable on which we could determine symptom related IFC changes, the low number of individuals in each category could have lead to inappropriate weighting of small amounts of data. Mitigating this somewhat is that participants with positive responses (improvement) showed IFC changes discrete from those who either did not change or who worsened, creating basically two functional groups-responders and non-responders. Supporting this pattern is that these IFC results were consistent with resting-state functional connectivity changes measured with BOLD, which also showed that those who improve with rTMS exhibit connectivity patterns different from those who either show no change or who worsen (Yuan et al. 2017).

Second, we did not use an active control for rTMS. This could have been helpful in determining random differences in IFC due to the passage of time. However, as we learned from a prior analysis, IFC measurements made pre- and post-rTMS without respect to symptom changes show no consistent patterns; they appeared to be random (Ding et al. 2014). Only IFC changes binned according to treatment response groups show any coherent relationship, as in our current analyses. In both these EEG and prior fMRI 
analyses, we required that IFC changes be directionally related to symptom changes, indicating that the study was internally controlled (if connectivity went up for one direction of symptom change, it was required to go down for the opposite symptom change). For ethical reasons, we could not give repetitive sessions of rTMS to healthy control participants in order to determine intrinsic long-term rTMS effects.

Third, no explicit correction of multiple comparisons in terms of the number of IC pairs and frequency bands was applied in the correlation analyses in steps two and three. Each IC represents signals from strongly synchronized neural activity generated by neural substrates covering distributed brain regions (Delorme et al. 2012). Therefore, the analyses on ICs automatically involve application of a spatial cluster-based correction for multiple comparisons (Maris and Oosternveld 2007), given that EEG signals at each channel reconstructed from each IC will have the same statistics as the IC statistic. However, it does not address correction for multiple comparisons across IC pairs and frequency bands. In future studies with more subjects, multiple comparisons correction should be implemented to further verify the identified patterns.

Finally, though the use of ICA has improved spatial specificity of neural activities compared to channel signal, it does not reveal detailed information about the identity of the underlying neural substrates that generate the signal. Advances in cortical source imaging techniques may better correlate electrophysiological signatures of IFCs with cortical structures (Yuan et al. 2016; Liao et al. 2012; Zhu et al. 2014; Ding 2009). In the current analyses, we were only able to identify the general region of the affected signal. However, symptom related long-range connectivity differences were sufficient to identify frequency specific fronto-parietal and interhemispheric connectivity changes relevant to symptom status that have already been informative in subsequent neuromodulation trials (NCT02540616).

\section{Conclusion}

Our study revealed the electrophysiological signatures of IFC underlying symptom changes induced by rTMS treatment in MdDS. Symptom improvement after rTMS was significantly correlated with IFCs that had predominantly reduced values in all frequencies with the notable exception of the low alpha band $(8-10 \mathrm{~Hz})$, in which IFC increased after successful treatment. A favorable response was associated with higher baseline IFC values and a greater coherence in the direction of IFC change induced by rTMS. Though this was true in all frequency bands, the strongest correlations were in the high alpha and beta bands. In addition, most of these IFCs covered frontal and parietal regions with long range and interhemispheric connectivity. Uncovering these prognostic and treatment related factors may aid in the development of more precise and effective neuromodulation therapies for motion perception disorders, including studies that aim to prime the brain by enhancing IFC and by targeting specific frequency bands for treatment.

Acknowledgements This work was supported by the Laureate Institute for Brain Research, the William K. Warren Foundation, an equipment and study grant from the MdDS Balance Disorders Foundation (YHC), The Springbank Foundation (YHC), an award through NSF EPSCoR RII Track-2 \#1539068 (LD, HY, and YHC), and NIH-NIHGMS grant P20GM121312 (YHC).

Author Contributions All authors were involved in the design, data collection, and/or analysis of the data. Manuscript draft was prepared by YHC and GS, and was reviewed and revised by all other authors.

\section{Compliance with Ethical Standards}

Conflict of interest The authors report no financial or ethical conflicts of interest in the execution of this study.

Open Access This article is distributed under the terms of the Creative Commons Attribution 4.0 International License (http://creativeco mmons.org/licenses/by/4.0/), which permits unrestricted use, distribution, and reproduction in any medium, provided you give appropriate credit to the original author(s) and the source, provide a link to the Creative Commons license, and indicate if changes were made.

\section{References}

Arroll M, Attree E, Cha YH, Dancey C (2014) The relationship between symptom severity, stigma, illness intrusiveness and depression in mal de debarquement syndrome (MdDS). J Health Psychol. https://doi.org/10.1177/1359105314553046

Bazanova OM, Vernon D (2014) Interpreting EEG alpha activity. Neurosci Biobehav Rev 44:94-110

Berger H (1929) Uber das Elektroencephalogramm des Menschen (On the human electroencephalogram). Archiv für Psychiatrie und Nervenkrankheiten

Bingham E, Hyvärinen A (2000) A fast fixed-point algorithm for independent component analysis of complex valued signals. Int J Neural Syst 10:1-8

Bisdorff A, Von Brevern M, Lempert T, Newman-Toker DE (2009) Classification of vestibular symptoms: towards an international classification of vestibular disorders. J Vestib Res 19:1-13

Brown JJ, Baloh RW (1987) Persistent mal de debarquement syndrome: a motion-induced subjective disorder of balance. Am J Otolaryngol 8:219-222

Buckner RL, Andrews-Hanna JR, Schacter DL (2008) The brain's default network: anatomy, function, and relevance to disease. Ann N Y Acad Sci 1124:1-38

Cha YH (2009) Mal de debarquement. Semin Neurol 29:520-527

Cha YH (2012) Less common neuro-otologic disorders. Continuum (Minneap Minn) 18:1142-1157

Cha YH (2015) Mal de debarquement syndrome: new insights. Ann N Y Acad Sci 1343:63-68

Cha YH, Cui Y (2013) Rocking dizziness and headache: a two-way street. Cephalalgia 33:1160-1169 
Cha YH, Brodsky J, Ishiyama G, Sabatti C, Baloh RW (2008) Clinical features and associated syndromes of mal de debarquement. J Neurol 255:1038-1044

Cha YH, Chakrapani S, Craig A, Baloh RW (2012) Metabolic and functional connectivity changes in mal de debarquement syndrome. PLoS ONE 7:e49560

Cha YH, Cui Y, Baloh RW (2013) Repetitive transcranial magnetic stimulation for mal de debarquement syndrome. Otol Neurotol $34: 175-179$

Cha YH, Urbano D, Pariseau N (2016) Randomized single blind sham controlled trial of adjunctive home-based tDCS after rTMS for mal de debarquement syndrome: safety, efficacy, and participant satisfaction assessment. Brain Stimul 9:537-544

Cha YH, Cui YY, Baloh RW (2018) Comprehensive clinical profile of mal de debarquement syndrome. Front Neurol. https://doi. org/10.3389/fneur.2018.00261

Chatrian GE, Petersen MC, Lazarter JA (1959) The blocking of the rolandic wicket rhythm and some central changes related to movement. Electroencephalogr Clin Neurophysiol 11:497-510

Chen JL, Ros T, Gruzelier JH (2013) Dynamic changes of ICA-derived EEG functional connectivity in the resting state. Hum Brain Mapp 34:852-868

Cohen R, Clarke AR, Hudspeth W, Barry RJ (2008) EEG power and coherence in autistic spectrum disorder. Clin Neurophysiol 119:1002-1009

Cooper NR, Croft RJ, Dominey SJ, Burgess AP, Gruzelier JH (2003) Paradox lost? Exploring the role of alpha oscillations during externally vs. internally directed attention and the implications for idling and inhibition hypotheses. Int J Psychophysiol 47:65-74

de Munck JC, Gonçalves SI, Huijboom L, Kuijer JP, Pouwels PJ, Heethaar RM, Lopes da Silva FH (2007) The hemodynamic response of the alpha rhythm: an EEG/fMRI study. Neuroimage 35:1142-151

Delorme A, Makeig S (2004) EEGLAB: an open source toolbox for analysis of single-trial EEG dynamics including independent component analysis. J Neurosci Methods 134:9-21

Delorme A, Palmer J, Onton J, Oostenveld R, Makeig S (2012) Independent EEG sources are dipolar. PLoS ONE 7:e30135

Dickson CT, Magistretti J, Shalinsky M, Hamam B, Alonso A (2000) Oscillatory activity in entorhinal neurons and circuits. Mechanisms and function. Ann N Y Acad Sci 911:127-150

Ding L (2009) Reconstructing cortical current density by exploring sparseness in the transform domain. Phys Med Biol 54:2683-2697

Ding L, Shou G, Yuan H, Urbano D, Cha YH (2014) Lasting modulation effects of rTMS on neural activity and connectivity as revealed by resting state EEG. IEEE Trans Biomed Eng 61:2070-2080

Donse L, Padberg F, Sack AT, Rush AJ, Arns M (2018) Simultaneous rTMS and psychotherapy in major depressive disorder: clinical outcomes and predictors from a large naturalistic study. Brain Stimul 11:337-345

Drysdale AT, Grosenick L, Downar J, Dunlop K, Mansouri F, Meng Y, Fetcho RN, Zebley B, Oathes DJ et al (2017) Resting-state connectivity biomarkers define neurophysiological subtypes of depression. Nat Med 23:28-38

Egorov AV, Hamam BN, Fransén E, Hasselmo ME, Alonso AA (2002) Graded persistent activity in entorhinal cortex neurons. Nature 420:173-178

Egorov AV, Unsicker K, von Bohlen, Halbach O (2006) Muscarinic control of graded persistent activity in lateral amygdala neurons. Eur J Neurosci 24:3183-3194

Engel AK, Fries P (2010) Beta-band oscillations-signalling the status quo? Curr Opin Neurobiol 20:156-165

Fischer AS, Keller CJ, Etkin A (2016) The clinical applicability of functional connectivity in depression: pathways toward more targeted intervention. Biol Psychiatry: Cogn Neurosci Neuroimaging 1:262-270

Fox MD, Buckner RL, White MP, Greicius MD, Pascual-Leone A (2012) Efficacy of transcranial magnetic stimulation targets for depression is related to intrinsic functional connectivity with the subgenual cingulate. Biol Psychiatry 72:595-603

Fransén E, Tahvildari B, Egorov AV, Hasselmo ME, Alonso AA (2006) Mechanism of graded persistent cellular activity of entorhinal cortex layer v neurons. Neuron 49:735-746

Golding JF (2016) Motion sickness. Handb Clin Neurol 137:371-390

Goldman RI, Stern JM, Engel J Jr, Cohen MS (2002) Simultaneous EEG and fMRI of the alpha rhythm. Neuroreport 13:2487

Gordon CR, Shupak A, Nachum Z, Hain TC (2000) Mal de debarquement. Arch Otolaryngol-Head Neck Surg 126:805-806

Hain TC, Cherchi M (2016) Mal de débarquement syndrome. Handb Clin Neurol 137:391-395

Hain TC, Hanna PA, Rheinberger MA (1999) Mal de debarquement. Arch Otolaryngol-Head Neck Surg 125:615-620

Klimesch W (1999) EEG alpha and theta oscillations reflect cognitive and memory performance: a review and analysis. Brain Res Rev 29:169-195

Klimesch W, Schimke H, Pfurtscheller G (1993) Alpha frequency, cognitive load and memory performance. Brain Topogr $5: 241-251$

Klimesch W, Doppelmayr M, Russegger H, Pachinger T, Schwaiger J (1998) Induced alpha band power changes in the human EEG and attention. Neurosci Lett 244:73-76

Klimesch W, Sauseng P, Hanslmayr S (2007) EEG alpha oscillations: the inhibition-timing hypothesis. Brain Res Rev 53:63-88

Kovacevic N, McIntosh AR (2007) Groupwise independent component decomposition of EEG data and partial least square analysis. Neuroimage 35:1103-1112

Lachaux JP, Rodriguez E, Martinerie J, Varela FJ (1999) Measuring phase synchrony in brain signals. Hum Brain Mapp 8:194-208

Laufs H, Krakow K, Sterzer P, Eger E, Beyerle A, Salek-Haddadi A, Kleinschmidt A (2003) Electroencephalographic signatures of attentional and cognitive default modes in spontaneous brain activity fluctuations at rest. Proc Natl Acad Sci USA 100:11053-11058

Liao C, Feng Z, Zhou D, Dai Q, Xie B, Ji B, Wang X, Wang X (2011) Dysfunction of fronto-limbic circuitry in depression. Neuroscience 201:231-238

Liao K, Zhu M, Ding L, Valette S, Zhang W, Dickens D (2012) Sparse imaging of cortical electrical current densities via wavelet transforms. Phys Med Biol 57:6881-6901

Liston C, Chen AC, Zebley BD, Drysdale AT, Gordon R, Leuchter B et al (2014) Default mode network mechanisms of transcranial magnetic stimulation in depression. Biol Psychiatry 76:517-526

Liu S, Sheng J, Li B, Zhang X (2017) Recent advances in non-invasive brain stimulation for major depressive disorder. Front Hum Neurosci 11:526

Mantini D, Perrucci MG, Del Gratta C, Romani GL, Corbetta M (2007) Electrophysiological signatures of resting state networks in the human brain. Proc Natl Acad Sci USA 104:13170-13175

Maris E, Oostenveld R (2007) Nonparametric statistical testing of EEG- and MEG-data. J Neurosci Methods 164:177-190

Mucci V, Canceri JM, Brown R, Dai M, Yakushin S, Watson S, Van Ombergen A, Topsakal V, Van de Heyning PH et al (2018) Mal de debarquement syndrome: a survey on subtypes, misdiagnoses, onset and associated psychological features. J Neurol 265:486-499

Mukamel R, Gelbard H, Arieli A, Hasson U, Fried I, Malach R (2005) Coupling between neuronal firing, field potentials, and FMRI in human auditory cortex. Science 309:951-954

Nachum Z, Shupak A, Letichevsky V, Ben-David J, Tal D, Tamir A, Talmon Y, Gordon CR, Luntz M (2004) Mal de debarquement and 
posture: reduced reliance on vestibular and visual cues. Laryngoscope 114:581-586

Neuper C, Pfurtscheller G (2001) Event-related dynamics of cortical rhythms: frequency-specific features and functional correlates. Int J Psychophysiol 43:41-58

Niedermeyer E (1990) Alpha-like rhythmical activity of the temporal lobe. Clin Electroencephalogr 21:210-224

Nolan H, Whelan R, Reilly RB (2010) FASTER: fully automated statistical thresholding for EEG artifact rejection. J Neurosci Methods 192:152-162

O'Reardon JP, Solvason HB, Janicak PG, Sampson S, Isenberg KE, Nahas Z, McDonald WM, Avery D, Fitzgerald PB, Loo C, Demitrack MA, George MS, Sackeim H (2007) Efficacy and safety of transcranial magnetic stimulation in the acute treatment of major depression: a multisite randomized controlled trial. Biol Psychiatry 62:1208-1216

Perera T, George MS, Grammer G, Janicak PG, Pascual-Leone A, Wirecki TS (2016) The Clinical TMS Society consensus review and treatment recommendations for TMS therapy for major depressive disorder. Brain Stimul 9:336-346

Raichle ME, MacLeod AM, Snyder AZ, Powers WJ, Gusnard DA, Shulman GL (2001) A default mode of brain function. Proc Natl Acad Sci 98:676-682

Ramkumar P, Parkkonen L, Hyvarinen A (2014) Group-level spatial independent component analysis of Fourier envelopes of restingstate MEG data. Neuroimage 86:480-491

Shou G, Ding L (2015) Detection of EEG spatial-spectral-temporal signatures of errors: a comparative study of ICA-based and channel-based methods. Brain Topogr 28:47-61

Shou G, Ding L, Dasari D (2012) Probing neural activations from continuous EEG in a real-world task: time-frequency independent component analysis. J Neurosci Methods 209:22-34

Shou G, Yuan H, Urbano D, Cha YH, Ding L (2014) Changes of symptom and EEG in mal de debarquement syndrome patients after repetitive transcranial magnetic stimulation over bilateral prefrontal cortex: a pilot study. Conf Proc IEEE Eng Med Biol Soc 42:94-97
Spitzer B, Haegens S (2017) Beyond the status quo: a role for beta oscillations in endogenous content (re)activation. eNeuro. https ://doi.org/10.1523/ENEURO.0170-17.2017

Stoffregen TA, Chen FC, Varlet M, Alcantara C, Bardy BG (2013) Getting your sea legs. PLoS ONE 8:e66949

Van Dijk KR, Hedden T, Venkataraman A, Evans KC, Lazar SW, Buckner RL (2009) Intrinsic functional connectivity as a tool for human connectomics: theory, properties, and optimization. J Neurophysiol 103:297-321

Van Diessen E, Numan T, Van Dellen E, Van Der Kooi AW, Boersma M, Hofman D, Van Lutterveld R, Van Dijk BW, van Straaten EC, Hillebrand A, Stam CJ (2015) Opportunities and methodological challenges in EEG and MEG resting state functional brain network research. Clin Neurophysiol 126:1468-1481

Whittingstall K, Logothetis NK (2009) Frequency-band coupling in surface EEG reflects spiking activity in monkey visual cortex. Neuron 64:281-289

Yuan H, Zotev V, Phillips R, Drevets WC, Bodurka J (2012) Spatiotemporal dynamics of the brain at rest-exploring EEG microstates as electrophysiological signatures of BOLD resting state networks. Neuroimage 60:2062-2072

Yuan H, Zotev V, Phillips R, Bodurka J (2013) Correlated slow fluctuations in respiration, EEG, and BOLD fMRI. Neuroimage 79:81-93

Yuan H, Ding L, Zhu M, Zotev V, Phillips R, Bodurka J (2016) Reconstructing large-scale brain resting-state networks from high-resolution EEG: spatial and temporal comparisons with fMRI. Brain Connect 6:122-135

Yuan H, Shou G, Urbano D, Ding L, Cha YH (2017) Resting state functional connectivity signature of treatment effects of rTMS in mal de debarquement syndrome. Brain Connect 7:617-626

Zhu M, Zhang W, Dickens DL, Ding L (2014) Reconstructing spatially extended brain sources via enforcing multiple transform sparseness. Neuroimage 86:280-293 